\title{
EFFECTS OF BULK AND GRAIN BOUNDARY RECOMBINATION ON THE EFFICIENCY OF COLUMNAR-GRAINED CRYSTALLINE SILICON FILM SOLAR CELLS
}

\author{
Michael G. Deceglie, Michael D. Kelzenberg, and Harry A. Atwater \\ Thomas J. Watson Laboratories of Applied Physics \\ California Institute of Technology, Pasadena, CA, USA
}

\begin{abstract}
Columnar-grained polycrystalline silicon films deposited at low temperatures are promising materials for use in thinfilm photovoltaics. We study the effects of recombination at grain boundaries, bulk intragranular recombination, grain size, and doping in such structures with twodimensional device physics simulations, explicitly modeling the full statistics and electrostatics of traps at the grain boundary. We characterize the transition from grainboundary-limited to bulk-lifetime-limited performance as a function of intergranular defect density and find that higher bulk lifetimes amplify grain boundary recombination effects in the intermediate regime of this transition. However, longer bulk lifetimes ultimately yield higher efficiencies. Additionally, heavier base doping is found to make performance less sensitive to grain boundary defect density.
\end{abstract}

\section{INTRODUCTION}

Polycrystalline silicon (poly-Si) thin-films synthesized by low-temperature locally epitaxial chemical vapor deposition on polycrystalline templates are attractive as absorber materials in next-generation thin-film solar cells. This microstructure, illustrated in Figure 1, maintains many of the benefits of thin-film photovoltaics associated with a$\mathrm{Si}$ and $\mu \mathrm{c}-\mathrm{Si}$ solar cells, but utilizes a higher-mobility absorber layer, thus relaxing the design requirements imposed by the $p$-i-n structure and realizing other performance benefits of crystalline absorber films.

This microstructure, and variants of it, can be achieved by locally epitaxial growth via e.g., hot-wire chemical vapor deposition (HWCVD) at temperatures below $500^{\circ} \mathrm{C}$ by introducing hydrogen along with silane during growth [1], and also by plasma-enhanced chemical vapor deposition (PECVD). Others have reported high quality epitaxial growth with HWCVD at higher substrate temperatures [2]. The low processing temperatures attainable with these methods are compatible with low-cost glass substrates and permit in situ hydrogen passivation, which is expected to mitigate grain boundary recombination.

For solar cells based on thin-film poly-Si absorber layers, it is critical to understand the impact of performance limiting factors such as bulk recombination within the crystalline grains, recombination at the grain boundary, and grain size. Previous work has considered the effects of these factors [3-9]; however, few studies [4,5] have thoroughly treated the effects of localized traps at grain boundaries.

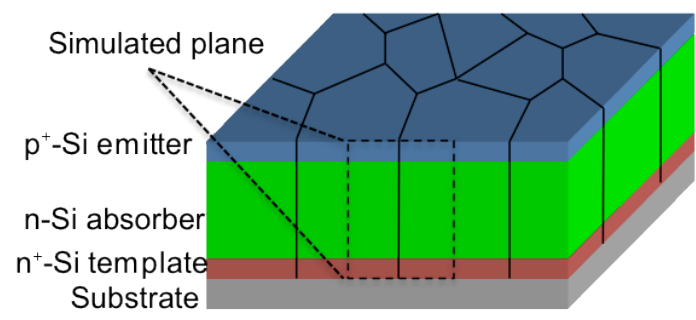

Figure 1: Illustration of the thin-film polycrystalline Si solar cell structure based on the use of local epitaxy to thicken a polycrystalline template depicting the two dimensional plane in which device performance is modeled.

Instead it is common to consider only effective recombination rates or surface recombination velocities, approximations which have been shown to be of limited use [5].

Here we develop a two-dimensional device model that explicitly simulates the full carrier statistics and electrostatics of traps at the grain boundary, along with other important physical processes including light absorption and bulk intragranular carrier recombination. We use this model to more thoroughly understand and optimize the device physics of polycrystalline Si thin-film solar cells.

\section{SIMULATION DETAILS}

The simulated structure consists of an n-type Si absorber layer of varying thickness and doping, a $50 \mathrm{~nm}$ p-type topside emitter doped at $10^{20} \mathrm{~cm}^{-3}$ and a $50 \mathrm{~nm}$ n-type layer on the back doped at $10^{20} \mathrm{~cm}^{-3}$ to provide a back surface field, both are assumed to be abrupt junctions. Contacts are modeled as ideal ohmic contacts. The simulation area is the width of one grain, with the grain boundary extending vertically through the center of the device, and Neumann boundary conditions at the edges, as illustrated in Figure 1. The grain width is varied from $50 \mathrm{~nm}-4 \mu \mathrm{m}$. To model the effect of bulk intragranular recombination, we vary the bulk Si Shockley-Read-Hall (SRH) recombination lifetime for electrons and holes. Doping dependent mobility is considered in all layers according to the model presented in Ref [10].

To model grain boundary recombination, we explicitly define the trap distribution in the band gap at the grain boundary. We model the energetic trap distribution at the grain boundary, $N(E)$, as a double exponential: 

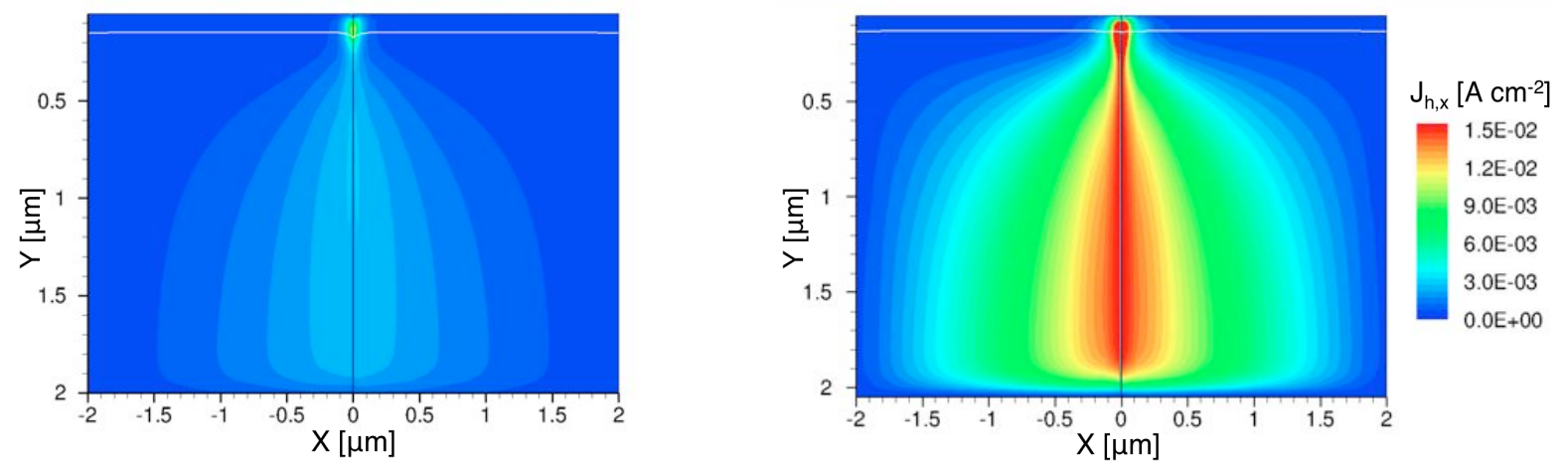

Figure 2: Simulation results showing the magnitude of the $x$-component of the hole current density in the base region under 1-sun illumination at open circuit for $4 \mu \mathrm{m}$ grain width, $2 \mu \mathrm{m}$ base thickness, $\mathrm{N}_{\mathrm{D}}=10^{16} \mathrm{~cm}^{-3}$ base doping level, and $\mathrm{N}_{\mathrm{gb}}=10^{14} \mathrm{~cm}^{-2} \mathrm{eV}^{-1}$, with bulk lifetimes of $10^{-8} \mathrm{~s}$ (left) and $10^{-6} \mathrm{~s}$ (right). The currents shown are directed toward the grain boundary. The white lines near the top of the plots indicate the depletion region edges.

$$
N(E)=N_{g b} e^{-E / \beta_{1}}+\gamma N_{g b} e^{-E / \beta_{2}}
$$

where $N_{g b}$ is a surface density of traps in units of $\mathrm{cm}^{-2} \mathrm{eV}^{-1}$ and $E$ is the energetic distance into the band gap from the corresponding band edge in $\mathrm{eV}$. In this work, the parameters used are: $\gamma=0.01, \beta_{1}=0.025 \mathrm{eV}$, and $\beta_{2}=$ $0.25 \mathrm{eV}$. We include this distribution for both a donor-like trap distribution distributed below the conduction band and an acceptor-like distribution above the valence band. Capture cross sections for both carrier types are taken as $10^{-15} \mathrm{~cm}^{2}[4,5]$. The value of $N_{g b}$ is varied to model the effect of grain boundary quality, including passivation effects.

We numerically simulate the performance of these structures using the Synopsis TCAD package including Sentaurus device physics simulator. The generation profile under illumination is calculated based on single pass absorption from a spectrally weighted sum of 95 light beams of different wavelength to approximate the AM1.5G solar spectrum. The carrier transport and Poisson equations are numerically solved on a finite-element mesh, varying the contact voltage to extract currentvoltage (IV) curves and photovoltaic performance parameters for each set of simulation parameters.

\section{RESULTS}

\section{Effects of bulk recombination and grain size}

We first characterize the transition from grain-boundarylimited to bulk-lifetime-limited performance as a function of the trap density at the grain boundaries and examine the device physics governing this transition. Figure 2 illustrates the interplay between bulk lifetime and grain boundary recombination. For identical grain size and grain boundary trap density, the longer bulk lifetime $\left(\tau=10^{-6} \mathrm{~s}\right)$ case exhibits a larger horizontal component of the minority current density directed toward the grain boundary as more carriers are driven to recombine at the boundary to meet the open circuit condition. We note that $\tau=10^{-6} \mathrm{~s}$ corresponds to a bulk minority carrier diffusion length of 33 $\mu \mathrm{m}$, and $\tau=10^{-8} \mathrm{~s}$ to a diffusion length of $3.3 \mu \mathrm{m}$. Even though $33 \mu \mathrm{m}$ is much longer than the width of the grain for the case pictured in Figure 2, the x-component of the current goes to 0 at $x= \pm 2 \mu \mathrm{m}$ due to the assumed presence in the model of another grain boundary at $x= \pm 4$ $\mu \mathrm{m}$.

The effects of these microscopic device physics on overall performance are presented in Figure 3. Toward the left of the plot in Figure 3a (corresponding to lower grain
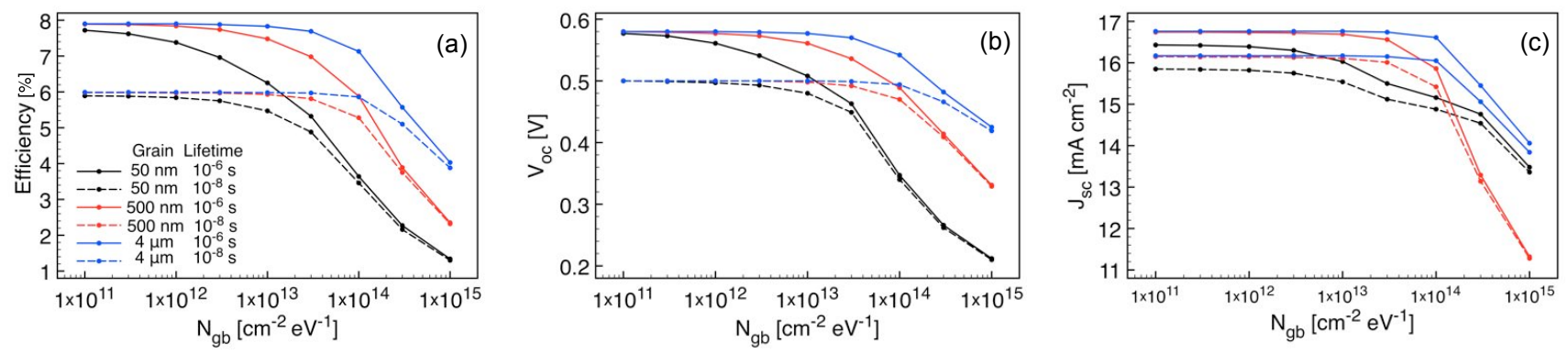

Figure 3: Simulated 1-sun photovoltaic performance parameters characterizing the transition between bulk SRH-limited performance at low intergranular defect density and grain-boundary-limited performance at high intergranular defect density for cells with $2 \mu \mathrm{m}$ thick base layers and $N_{D}=10^{16} \mathrm{~cm}^{-3}$. (a) Efficiency, (b) $V_{o c}$, (c) short-circuit current density. 
boundary trap densities), efficiencies for the same bulk lifetimes converge with one another and become much less sensitive to grain size, indicating bulk-lifetime-limited performance. Toward the right of the plot (corresponding to higher grain boundary trap densities), curves of the same grain size converge, indicating grain-boundarylimited performance. We also see that, in the intermediate regime between grain-boundary-dominated and bulkdominated performance, improved bulk lifetimes augment the reduction in efficiency due to reduced grain size (also evident in Figure 2). These results emphasize the importance of long bulk lifetimes and the inherent trade-off between grain size and grain boundary passivation. In Figure 3c, we note that the $50 \mathrm{~nm}$ grain, high trap density cases actually produce higher short circuit currents than the $500 \mathrm{~nm}$ grain cases. We attribute this to significant depletion of the small grains resulting in the onset of carrier collection by drift. However, the open circuit voltage is so strongly degraded in this case that the ultimate efficiency remains lower than for larger grain microstructures.

\section{Effects of base doping}

Next, we investigate the effect of base doping level on recombination at the grain boundary. Figure 4 shows that when the base is more lightly doped, the partially depleted
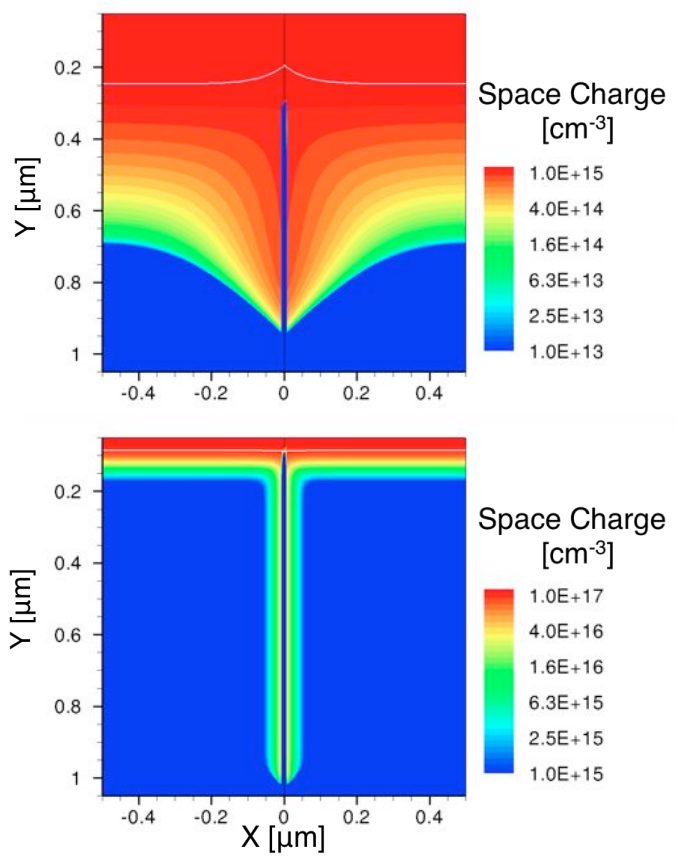

Figure 4: Simulation results showing partial depletion in the base surrounding the grain boundary under 1-sun at open circuit for bases doped at $N_{D}=10^{15} \mathrm{~cm}^{-3}$ (top) and $10^{17} \mathrm{~cm}^{-3}$ (bottom). The color scales extend from $1 \%$ to $100 \%$ of the base doping level. The base is $1 \mu \mathrm{m}$ thick, the grain is $1 \mu \mathrm{m}$ wide, $\tau=10^{-6} \mathrm{~s}$, and $\mathrm{N}_{\mathrm{gb}}=10^{14} \mathrm{~cm}^{-2} \mathrm{eV}^{-1}$.

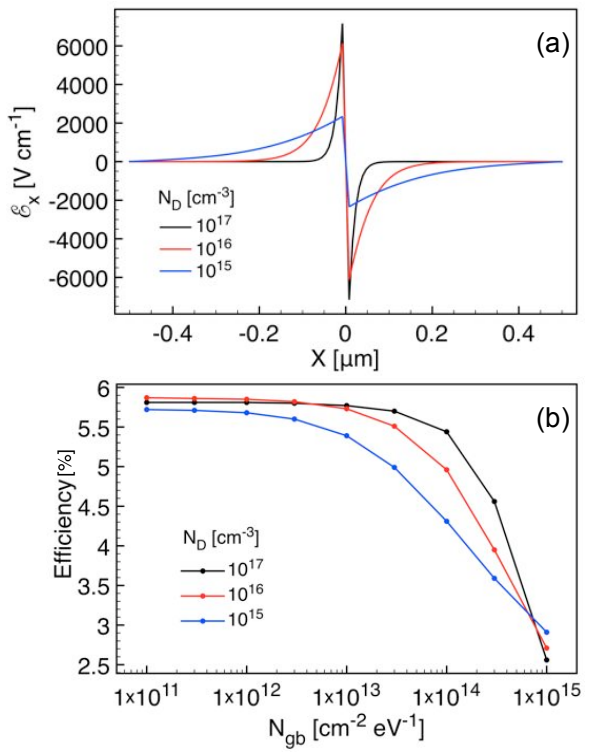

Figure 5: Simulation results for devices under 1-sun illumination with a $1 \mu \mathrm{m}$ thick base, $1 \mu \mathrm{m}$ wide grain, and $\tau=10^{-6} \mathrm{~s}$. (a) The $\mathrm{x}$-component of the electric field along the horizontal slice at the middle of the base region $(y=550 \mathrm{~nm})$ for $\mathrm{N}_{\mathrm{gb}}=10^{14} \mathrm{~cm}^{-2} \mathrm{eV}^{-1}$ and selected base doping levels at open circuit. Note that the field is directed toward the grain boundary and extends further into the bulk with decreasing base doping level. (b) Cell performance for varying base doping level as grain boundary quality is reduced.

region surrounding the grain boundary extends further into the bulk. This extended partially depleted region is characterized by an electric field directed toward the grain boundary (as indicated in Figure $5 \mathrm{a}$ ) that drives minority carrier current flow toward the boundary under open circuit conditions. We also note that our model, which explicitly considers trap electrostatics at the grain boundary, provides a detailed view of the microscopic device physics that would not be evident in analysis relying on the assumption of quasineutrality.

In agreement with this physical understanding, Figure $5 \mathrm{~b}$ confirms that the efficiencies of the simulated devices with higher base doping levels are more robust with respect to increasing grain boundary trap density. This agrees with previous reports $[5,6]$. Based on the device physics described above and illustrated in Figure 4, we attribute the increased performance of the more heavily doped devices to the shorter Debye screening length, which confines the electric field to the region immediately surrounding the grain boundary. Although even higher doping might further reduce grain boundary recombination, such devices would ultimately display degraded performance due to reduced bulk minority carrier lifetime at high doping, reduced efficacy of the back surface field, and degraded carrier mobility. We also note that regions of heavier doping surrounding the grain boundary would likely have beneficial impact, since they 
would limit the physical extent of the partially depleted region. This case is often observed in practice since dopant segregation is known occur at grain boundaries.

\section{CONCLUSION}

A numerical device physics model for polycrystalline Si columnar-grained thin-film solar cells enables us to understand effects of key material quality parameters on device performance. By explicitly modeling the carrier statistics and electrostatics of traps at grain boundaries, we avoid the need to employ simplifying assumptions such as quasineutrality or an effective diffusion length. This provides insight into the operation of these solar cells, and suggests routes towards enhancing their efficiency, in addition to passivation and grain enlargement, by engineering doping profiles to mitigate the effects of grainboundary recombination. These modeling techniques will be useful in designing future polycrystalline Si thin-film photovoltaics, and will benefit from improved understanding of surface and bulk trap distributions and doping profiles within these materials.

\section{ACKNOWLEDGEMENTS}

The authors wish to thank Daniel B. Turner-Evans for useful discussions. This work is supported by BP Solar.

\section{REFERENCES}

[1] C.E. Richardson, K. Langeland, and H.A. Atwater, "Electronic properties of low temperature epitaxial silicon thin film photovoltaic devices grown by HWCVD", Thin Sol. Films 516, 2008, pp. 597-599.

[2] Q. Wang et al., "Significant improvement in silicon chemical vapor deposition epitaxy above the surface dehydrogenation temperature", J. Appl. Phys. 100, 2006, 093520 .

[3] J.H. Werner et al., "From polycrystalline to single crystalline silicon on glass", Thin Sol. Films 383, 2001, pp. 95-100.

[4] P.P. Altermatt and G. Heiser, "Predicted electronic properties of polycrystalline silicon from three-dimensional device modeling combined with defect-pool model", J. Appl. Phys. 92, 2002, pp. 2561-2574.

[5] S.A. Edmiston et al., "Improved modeling of grain boundary recombination in bulk and $p-n$ junction regions of polycrystalline silicon solar cells", J. Appl. Phys. 80, 1996, pp. 6783-6795.

[6] E. Christoffel et al., "A two-dimensional modeling of the fine-grained polycrystalline silicon thing-film solar cells", Thin Sol. Films 403, 2002, pp. 258-262.

[7] T. Fujisaki, A. Yamada, and M. Konagai, "Effects of grain boundaries on cell performance of poly-silicon thin film solar cells by 2-D simulation", Sol. Energ. Mat. Sol. C. 74, 2002, pp. 331-337.

[8] H. Takakura and Y. Hamakawa, "Device simulation and modeling of microcrystalline silicon solar cells", Sol. Energ. Mat. Sol. C. 74, 2002, pp. 479-487.

[9] K. Kurobe et al., "Effects of grain boundaries in polycrystalline silicon thin-film solar cells based on the two-dimensional model", Sol. Energ. Mat. Sol. C. 65, 2001, pp. 201-209.

[10] G. Masetti, M. Severi, and S. Solmi, "Modeling of carrier mobility against carrier concentration in arsenic-, phosphorus-, and boron-doped silicon", IEEE T. Electron. Dev. 30, 1983, pp. 764-769. 